\title{
Evaluating the Accuracy of the VitalWellness Device
}

Nicole Polanco ${ }^{1,2^{*}}$, BA; Sharon Odametey ${ }^{1,2,3^{*}}$, MPH; Neda Derakhshani ${ }^{1,2^{*}}$, MSc (Comp Sci); Mark Khachaturian ${ }^{4 *}$, $\mathrm{PhD}$; Connor Devoe ${ }^{1,2^{*}}$, BS; Kamal Jethwani ${ }^{1,2,3^{*}}$; Sujay Kakarmath ${ }^{1,2,3^{*}}$, MD, MS

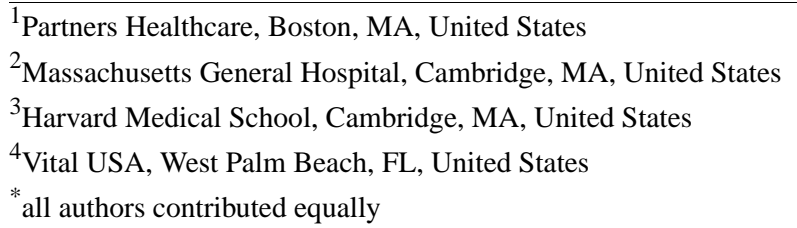

\section{Corresponding Author:}

Nicole Polanco, BA

Partners Healthcare

25 New Chardon St

Boston, MA

United States

Phone: 6177243152

Email: nvpolanco@partners.org

\begin{abstract}
Background: Wellness devices for health tracking have gained popularity in recent years. Additionally, portable and readily accessible wellness devices have several advantages when compared to traditional medical devices found in clinical environments The VitalWellness device is a portable wellness device that can potentially aide vital sign measuring for those interested in tracking their health.
\end{abstract}

Objective: In this diagnostic accuracy study, we evaluated the performance of the VitalWellness device, a wireless, compact, non-invasive device that measures four vital signs (blood pressure (BP), heart rate (HR), respiratory rate (RR), and body temperature using the index finger and forehead.

Methods: Volunteers age $\geq 18$ years were enrolled to provide blood pressure (BP), heart rate (HR), respiratory rate (RR), and body temperature. We recruited participants with vital signs that fell within and outside of the normal physiological range. A sub-group of eligible participants were asked to undergo an exercise test, aerobic step test and/or a paced breathing test to analyze the VitalWellness device's performance on vital signs outside of the normal physiological ranges for HR and RR. Vital signs measurements were collected with the VitalWellness device and FDA-approved reference devices. Mean, standard deviation, mean difference, standard deviation of difference, standard error of mean difference, and correlation coefficients were calculated for measurements collected; these measurements were plotted on a scatter plot and a Bland-Altman plot. Sensitivity analyses were performed to evaluate the performance of the VitalWellness device by gender, skin color, finger size, and in the presence of artifacts.

Results: 265 volunteers enrolled in the study and 2 withdrew before study completion. Majority of the volunteers were female $(62 \%)$, predominately white $(63 \%)$, graduated from college or post college $(67 \%)$, and employed (59\%). There was a moderately strong linear relationship between VitalWellness BP and reference BP $(\mathrm{r}=0.7, P<.05)$ and VitalWellness RR and reference RR measurements $(\mathrm{r}=0.7, P<.05)$. The VitalWellness HR readings were significantly in line with the reference HR readings $(\mathrm{r}=0.9$, $P<.05)$. There was a weaker linear relationship between VitalWellness temperature and reference temperature $(\mathrm{r}=0.3, P<.05)$. There were no differences in performance of the VitalWellness device by gender, skin color or in the presence of artifacts. Finger size was associated with differential performance for RR.

Conclusions: Overall, the VitalWellness device performed well in taking BP, HR, and RR when compared to FDA-approved reference devices and has potential serve as a wellness device. To test adaptability and acceptability, future research may evaluate user's interactions and experiences with the VitalWellness device at home. In addition, the next phase of the study will evaluate transmitting vital sign information from the VitalWellness device to an online secured database where information can be shared with HCPs within seconds of measurement.

(iproc 2019;5(1):e16250) doi: $\underline{10.2196 / 16250}$ 


\section{KEYWORDS}

personal tracking; vital signs; blood pressure; heart rate; home monitoring

Edited by J Brown; this is a non-peer-reviewed article. Submitted 13.09.19; accepted 13.09.19; published 02.10.19.

Please cite as:

Polanco N, Odametey S, Derakhshani N, Khachaturian M, Devoe C, Jethwani K, Kakarmath $S$

Evaluating the Accuracy of the VitalWellness Device

iproc 2019;5(1):e16250

URL: http://www.iproc.org/2019/1/e16250/

doi: $\underline{10.2196 / 16250}$

PMID:

CNicole Polanco, Sharon Odametey, Neda Derakhshani, Mark Khachaturian, Connor Devoe, Kamal Jethwani, Sujay Kakarmath. Originally published in Iproceedings (http://www.iproc.org), 02.10.2019 This is an open-access article distributed under the terms of the Creative Commons Attribution License (https://creativecommons.org/licenses/by/4.0/), which permits unrestricted use, distribution, and reproduction in any medium, provided the original work, first published in Iproceedings, is properly cited. The complete bibliographic information, a link to the original publication on http://www.iproc.org/, as well as this copyright and license information must be included. 